\title{
ON-LINE SIGNATURE PARTITIONING USING A POPULATION BASED ALGORITHM
}

\author{
Marcin Zalasiński ${ }^{1, *}$, Krystian Łapa ${ }^{1}$, Krzysztof Cpałka ${ }^{1}$, \\ Krzysztof Przybyszewski ${ }^{2}$, Gary G. Yen ${ }^{3}$ \\ ${ }^{1}$ Czestochowa University of Technology, \\ Department of Computational Intelligence, Poland \\ ${ }^{2}$ Information Technology Institute, University of Social Sciences, Łódź, Poland \\ Clark University, Worcester, MA 01610, USA \\ ${ }^{3}$ School of Electrical and Computer Engineering, \\ Oklahoma State University, Stillwater, OK 74075 USA \\ *E-mail:marcin.zalasinski@pcz.pl
}

Submitted: 19th September 2019; Accepted: 15th November 2019

\begin{abstract}
The on-line signature is a biometric attribute which can be used for identity verification. It is a very useful characteristic because it is commonly accepted in societies across the world. However, the verification process using this particular biometric feature is a rather difficult one. Researchers working on identity verification involving the on-line signature might face various problems, including the different discriminative power of signature descriptors, the problem of a large number of descriptors, the problem of descriptor generation, etc. However, population-based algorithms (PBAs) can prove very useful when resolving these problems. Hence, we propose a new method for on-line signature partitioning using a PBA in order to improve the verification process effectiveness. Our method uses the Differential Evolution algorithm with a properly defined evaluation function for creating the most characteristic partitions of the dynamic signature. We present simulation results of the proposed method for the BioSecure DS2 database distributed by the BioSecure Association.
\end{abstract}

Keywords: on-line signature, biometrics, signature partitioning, population-based algorithm

\section{Introduction}

The signature is a commonly used and generally socially acceptable form of authorization. It is also a characteristic used in behavioral biometrics for identity verification $[16,25]$. The on-line signature (called also the dynamic signature or DS) is a special kind of this characteristic which also contains information about the dynamics of a signing process (e.g. pen pressure, instant pen velocity, pen tilt angle, etc.) in the form of signals changing over time [19]. These signals describing the signature are acquired when a digital input device is being used, e.g. a graphic tablet. The DS analysis allows us to obtain many pieces of information characteristic of an individual signer, which makes the verification process more effective than in the case of using a so-called static signature which only contains information about the shape of a signature $[6,7]$. 
The DS is a very interesting biometric attribute, which can be very useful when put to practice. In the literature, there are a few groups of methods used for the on-line signature verification $[8,9,13]$. One of them is the regional approach based on the descriptors created in specific regions (partitions) of the signature. The descriptors are determined during the training session of the biometric system, and next the template describing an individual signer is created using their values. During the identity verification phase the values of descriptors obtained from the test signature are compared to the template and the system makes a decision whether the identity has been verified correctly or not.

However, identity verification using the DS is an issue that still has a number of open problems, which include the problem of the different discriminative power of signature descriptors, the problem of a large number of descriptors describing a specific biometric feature, the problem of an effective personalization of descriptors, the problem of a scalability of solutions, etc.

An effective tool supporting identity verification using the DS are e.g. PBAs [17]. Most PBAs are based on nature or specific phenomena occurring in nature (see e.g. [15, 18]). Such algorithms iteratively process the population of candidate solutions (individuals) trying to improve them with regard to the quality of a given objective. This type of procedure causes that these algorithms can be used to solve many problems (mainly optimization), and therefore they are referred to as metaheuristics. At the same time these algorithms do not guarantee finding the optimal solution, but they make it possible to find very good solutions within an acceptable time. In our previous works, we used PBAs to select a subset of descriptors describing the DS [24] and to predict changes in these descriptors [23].

In this paper we propose a new method for creating the most characteristic partitions of the signature so as to improve the effectiveness of the verification process. It uses the capabilities offered by PBAs to efficiently generate boundaries of the partitions containing signature descriptors. In the solutions used so far $[13,3]$, these boundaries have been determined using basic mathematical operators such as e.g. the arithmetic average. Meanwhile, the proper generation of descriptors (implemented independently for each user) is a key issue in terms of the effectiveness of signature verification. Therefore, using PBAs to generate descriptors seems to be the right approach, but it requires adaptation to the specifics of the descriptor processing methods and proper integration with these methods. An example solution of this particular problem is presented in this work.

Effectiveness of the proposed partitioning method is evaluated with the use of a flexible fuzzy one-class classifier, which, among others, we proposed in [4]. This classifier does not require a classic learning process and its parameters are determined analytically.

This paper is organized into five sections. Section 2 contains a description of the classic partitioning approach. Section 3 presents a description of the population-based approach proposed in this paper. Section 4 presents the simulation results, and Section 5 contains the conclusions.

\section{Classic approach to on-line sig- nature partitioning}

In the literature we can find several approaches to the DS partitioning including the following three:

- Horizontal partitioning [3]. This is a method of the DS partitioning which assumes the division of the signals describing dynamics of the signing process (pen velocity, pen pressure, pen angle, etc.) into two horizontal parts. In [3] the division is performed on the basis of the velocity and pressure signals' values. Partitions created in this approach contain information about similarity of the signature trajectories in the regions associated with high and low pen velocity and pressure.

- Vertical partitioning [2]. This is a method of the DS partitioning which assumes the division of the signals describing dynamics of the signing process into a number of vertical parts. The division is performed on the basis of the time step values of the signature signals. Partitions created in this approach contain information about similarity of the signature trajectories in the regions associated with the phase of signature creation.

- Hybrid partitioning [4]. This is a method of the 
DS partitioning which assumes the division of the signals describing dynamics of the signing process into many vertical sections on the basis of the time step values of the signature signals. Next, in each section the signals describing the signature are divided into two horizontal sections on the basis of the velocity and pressure signals' values, creating partitions. Partitions created in this approach contain information about the similarity of the signature trajectories in the regions associated with the phase of signature creation and also associated with high and low pen velocity and pressure.

In this paper, we focus on the horizontal partitioning approach. Partitioning results from creating the signature descriptors. They are evaluated by the method of their verification and determined using the following steps:

1. Preprocessing. First, reference signatures of the user should be pre-processed by commonly used methods (see e.g. $[10,13]$ ) in order to match their length, rotation, scale and offset. This step is performed in a similar way in the test phase for a test signature.

2. Creation of partitions. In this step horizontal partitions are created. Partitions indicated by the elements of vector $\mathbf{p}_{i}^{\{s\}}=\left[p_{i, k=1}^{\{s\}}, p_{i, k=2}^{\{s\}}, \ldots, p_{i, k=K_{i}}^{\{s\}}\right]$ are determined as follows

$$
p_{i, k}^{\{s\}}=\left\{\begin{array}{l}
1 \quad \text { for } \quad s_{i, j=j \text { Base }, k}<a v g_{i}^{\{s\}} \\
2 \text { for } \quad s_{i, j=j \text { Base }, k} \geq a v g_{i}^{\{s\}}
\end{array}\right.
$$

where $i$ is the user index, $j$ is the index of the signature, $k$ is the index of the signal sample, $K_{i}$ is the number of signal samples, $s$ indicates velocity $v$ or pressure $z$ signal (the set of signals considered can be changed as needed), jBase is the index of the base signature which is the training signature most similar to the others, avg is the average value of velocity or pressure signal. This step is performed only in the learning phase for the reference signatures.

3. Creation of the template. The templates of the signatures are averaged fragments of the reference signatures represented by the shape trajectories. They are indicated by the elements of vector $\mathbf{t c}_{i, p}^{\{s, a\}}=\left[t c_{i, p, k=1}^{\{s, a\}}, t c_{i, p, k=2}^{\{s, a\}}, \ldots, t c_{i, p, k=K c_{i, p}\{s\}}^{\{s, a\}}\right]$, where $K c_{i, p}^{\{s\}}$ is the number of samples in partition $p$ of user $i$, associated with trajectory signal $a$ ( $x$ or $y$ ), created on the basis of signal $s$. These elements are determined as follows

$$
t c_{i, p, k}^{\{s, a\}}=\frac{1}{J} \sum_{j=1}^{J} a_{i, j, p, k}^{\{s\}},
$$

where $\mathbf{a}_{i, j, p}^{\{s\}}=\left[a_{i, j, p, k=1}^{\{s\}}, \ldots, a_{i, j, p, k=K c_{i, p}^{\{s\}}}^{\{s\}}\right]$ is a trajectory of reference signature $j$ of user $i$ created on the basis of signal $s$, which belongs to partition $p$. This step is performed only in the learning phase.

4. Creation of the descriptors. In this step descriptors $d_{i, p}^{\{s, a\}}$ of signature $j$ are created in the following way

$$
d_{i, j, p}^{\{s, a\}}=\sqrt{\sum_{k=1}^{K c_{i, p}^{\{s\}}}\left(t c_{i, p, k}^{\{s, a\}}-a_{i, j, p, k}^{\{s\}}\right)^{2}} .
$$

This step is performed in a similar way in the test phase for a test signature.

We can see that in the classic method for the DS partitioning, average values of trajectories are used to divide a signature into partitions. However, in this solution it is not guaranteed that the performed division allows us to obtain the most characteristic descriptors from an individual user. Due to this, we propose a population based approach to divide the signature into partitions characteristic of a given user. Our approach is described in the next Section.

\section{Population based approach to on-line signature partitioning}

The idea of the DS verification method using evolutionary horizontal partitioning is presented in Figure 1.

In order to specify the partition boundaries (division points) used, you create partitions for the individual user. We use the population-based Differential Evolution (DE, [5]) algorithm with a proper defined evaluation function. The details of this process can be found in the following part of this Section. 
population based algorithm

for creating horizontal partitions

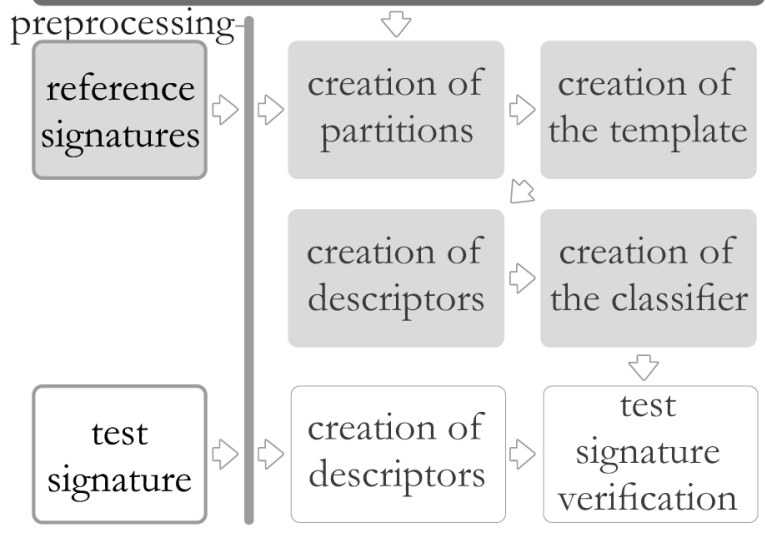

Figure 1. Structure of the method for the DS verification using $\mathrm{z}$ evolutionary horizontal partitioning

\subsection{Description of the DE algorithm}

The DE algorithm is an optimization method from the group of evolutionary algorithms and these belong to the PBA group.

The DE starts searching from initialization of the population (Figure 2) in which each of Npop individuals is encoded with the parameters randomly distributed in the so-called search space (the space limiting the allowed parameter values, which arises from the considered problem specification). Then, each individual in the population is evaluated using the evaluation function (also called the objective function) also associated with the problem under consideration. This process is followed by the iterative processing of the population, repeated until a specific iteration number is achieved or a satisfactory result is found. In each iteration for each individual a clone is created, which is modified according to the following formula

$$
X_{c h, g}^{\prime}=\left\{\begin{array}{l}
X_{l, g}+F \cdot\left(X_{m, g}-X_{n, g}\right) \\
\text { for rand }<C R \text { or } g=R \\
X_{c h, g} \text { otherwise, }
\end{array}\right.
$$

where $\mathbf{X}_{c h}$ are individuals, $\mathbf{X}_{c h}^{\prime}$ are individual clones, $c h=1, \ldots, N$ pop are indexes of individuals, $g=1, \ldots, D$ are indexes of individuals parameters $(D$ is the number of parameters being optimized), $F \in\langle 0 ; 2\rangle$ is differential weight, $C R \in\langle 0 ; 1\rangle$ is crossover probability, $\{l, m, n\}$ are indexes of randomly selected individuals from the population (without repetition and different than $c h$ ), $R$ is a randomly selected integer from set $\{1, D\}$ (thus always one individual parameter is modified), rand is a random function that returns values from range $\langle 0 ; 1\rangle$. Each clone is repaired (parameters are narrowed to search space) and evaluated. If the clone has a better value of the objective function than its parent, then it replaces the parent. After the iteration or interruption of the algorithm, the best solution found is presented. The block schema of the algorithm is presented in Figure 2.

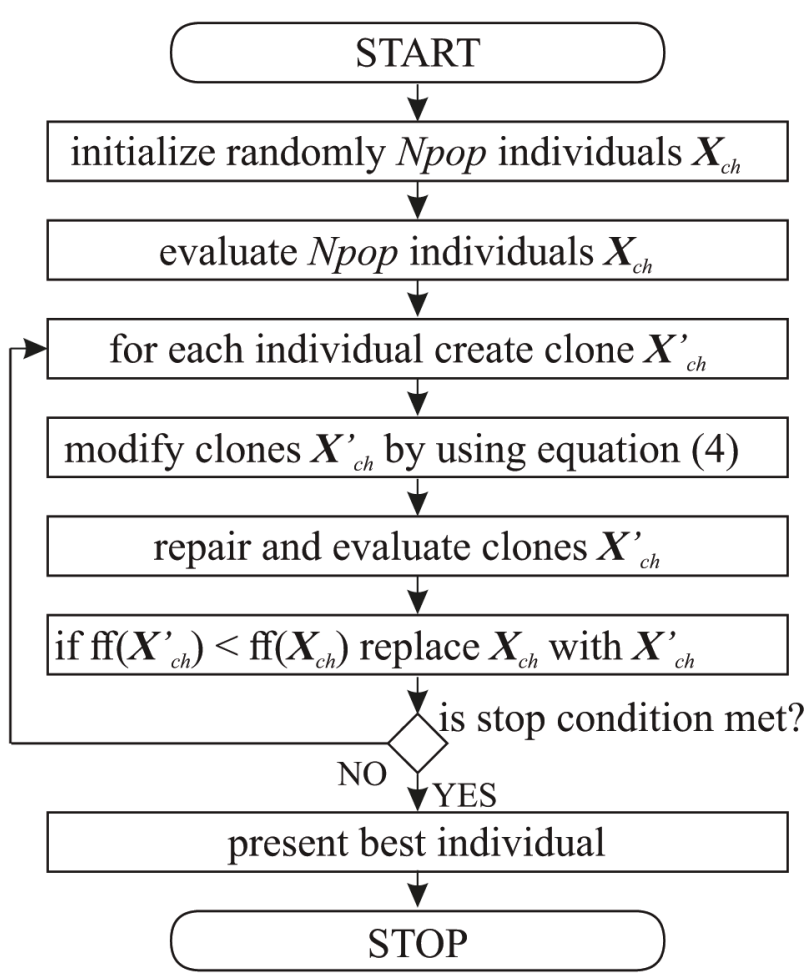

Figure 2. Block schema of the Differential Evolution algorithm

\subsection{Description of the proposed method}

In the proposed method the PBA is used for selecting the division points values $d i v_{i}^{\{s\}}$ used for creating signature partitions. These values replace average values $a v g_{i}^{\{s\}}$ of signal $s=v$ or $s=z$ presented in equation (1):

$$
p_{i, k}^{\{s\}}=\left\{\begin{array}{lll}
1 & \text { for } \quad & s_{i, j=j B a s e, k}<d i v_{i}^{\{s\}} \\
2 & \text { for } \quad & s_{i, j=j B a s e, k} \geq d i v_{i}^{\{s\}}
\end{array} .\right.
$$

In the proposed method each individual $\mathbf{X}_{i, c h}$ from the population encodes two division points of 
the signature determined for user $i$. It is represented as follows

$$
\mathbf{X}_{i, c h}=\left\{d i v_{i}^{\{v\}}, d i v_{i}^{\{z\}}\right\}=\left\{X_{1, c h}, X_{2, c h}\right\}
$$

The algorithm aims at selecting a subset of the values of division points used for creating partitions of the signature which contain the most characteristic signature descriptors of user $i$.

Evaluation of the population of individuals is performed using a specially designed fitness function (ff). The value of $\mathrm{ff}$ is determined using descriptor values from the partitions created on the basis of the values encoded in the individual. The function's value also takes into account a ratio between the number of discretization points in the signature partitions associated with the same signal $s$ because we assume that the size of partition should not be too small. In this case the information about the signature represented by this partition should not be considered credible.

First, in order to compute the value of ff we have to calculate the values of parameters $R p_{i, j}^{\{s\}}$, which indicate a ratio between the number of discretization points in signature partitions associated with the same signal $s$. It is determined as follows

$$
R p_{i, j}^{\{s\}}=\left\{\begin{array}{l}
1-\frac{K c_{i, 1}^{\{s\}}}{K s_{i, 0}^{s\}}} \text { for } K c_{i, 0}^{\{s\}} \geq K c_{i, 1}^{\{s\}} \\
1-\frac{K c_{i, 0}^{s\}}}{K c_{i, 1}^{s\}}} \text { otherwise }
\end{array}\right.
$$

Next, we have to normalize the values of descriptors representing similarity of the training signatures to the template. The normalization is performed using membership function $\mu\left(d_{i, j, p}^{\{s, a\}}\right)$ defined as follows

$$
\mu\left(d_{i, j, p}^{\{s, a\}}\right)=\frac{1}{1+\exp \left(5-2 \cdot d_{i, j, p}^{\{s, a\}}\right)} .
$$

Next, we averaged the values of the above mentioned variables in the context of each training signature $j$ of considered user $i$. After this step, we obtained the values of parameters $\operatorname{avg} D_{i, j}$ and $\operatorname{avg} R_{i, j}$ defined as follows

$$
\left\{\begin{array}{l}
\operatorname{avg} D_{i, j}=\frac{\left(\begin{array}{c}
\mu\left(d_{i, j, 0}^{\{v, x\}}\right)+\mu\left(d_{i, j, 1}^{\{v, x\}}\right)+\ldots \\
+\mu\left(d_{i, j, 0}^{\{z, y\}}\right)+\mu\left(d_{i, j, 1}^{\{z, y\}}\right)
\end{array}\right)}{4 \cdot p} \\
\operatorname{avg} R_{i, j}=\frac{1}{2} \cdot\left(R p_{i, j}^{\{v\}}+R p_{i, j}^{\{z\}}\right)
\end{array}\right.
$$

where $\operatorname{avg} D_{i, j}$ is the average value of descriptors in the context of signature $j$ and $a v g R_{i, j}$ is the average value of parameters $R p_{i, j}^{\{s\}}$ in the context of signature $j$.

Next, the value of the fitness function is calculated using weighted algebraic triangular norm $T^{*}\{\cdot\}[1]$

$$
\begin{aligned}
& \operatorname{ff}\left(\mathbf{X}_{i, c h}\right)= \\
& =T^{*}\left\{\begin{array}{c}
\operatorname{avg} D_{i, 1}, \ldots, \operatorname{avg} D_{i, J}, \\
\operatorname{avg} R_{i, 1}, \ldots, a v g R_{i, J} \\
w D_{i, 1}, \ldots, w D_{i, J} \\
w R_{i, 1}, \ldots, w R_{i, J}
\end{array}\right\}= \\
& =\left(\begin{array}{c}
\left(1-w D_{i, 1} \cdot\left(1-\operatorname{avg} D_{i, 1}\right)\right) \cdot \ldots \\
\cdot\left(1-w R_{i, J} \cdot\left(1-\operatorname{avg} R_{i, J}\right)\right)
\end{array}\right),
\end{aligned}
$$

where t-norm $T^{*}\{\cdot\}$ is a generalization of the usual two-valued logical conjunction (studied in the classical logic), $w D_{i, j} \in[0,1]$ and $w R_{i, j} \in[0,1]$ are the weights of importance of arguments $\operatorname{avg} D_{i, j}$ and $\operatorname{avg} R_{i, j}$.

The purpose of the DE algorithm is a maximization of the proposed fitness function. When the algorithm finishes its work, the values of division points $d i v_{i}^{\{v\}}$ and $d i v_{i}^{\{z\}}$ are obtained. Next, the signature is partitioned using these values, and the templates and descriptors of the signature are created (see Section 2). After this process, the flexible fuzzy one-class classifier is determined. In this paper we use one of the classifiers which we proposed in [4], which offers its detailed description. The most important feature of the classifier is that it does not require a learning process.

\section{Simulations}

Assumptions on the simulations adopted and carried out under the present research can be summarized as follows

- The simulations were performed using the 
Table 1. Comparison of the accuracy of the method using the on-line signature partitioning with the population-based algorithm to other methods for the DS verification using the BioSecure database.

\begin{tabular}{lllll}
\hline \hline Id. & Method & Average FAR & Average FRR & Average error \\
\hline 1. & $\begin{array}{l}\text { Different methods presented } \\
\text { in [12] }\end{array}$ & - & $3.48 \%-30.13 \%$ \\
\hline 2. & $\begin{array}{l}\text { Method using horizontal par- } \\
\text { titioning presented in [3] }\end{array}$ & $2.94 \%$ & $4.45 \%$ & $3.70 \%$ \\
\hline 3. & $\begin{array}{l}\text { Method using vertical parti- } \\
\text { tioning presented in [2] }\end{array}$ & $3.12 \%$ & $4.15 \%$ & $3.64 \%$ \\
\hline 5. & $\begin{array}{l}\text { Method using hybrid parti- } \\
\text { tioning presented in [4] }\end{array}$ & $3.36 \%$ & $3.30 \%$ & $3.33 \%$ \\
\hline 4. & Our method & $\mathbf{2 . 9 2 \%}$ & $\mathbf{3 . 2 4} \%$ & $\mathbf{3 . 0 8 \%}$ \\
\hline \hline
\end{tabular}

BioSecure dynamic signature database DS2 [11] which contains signatures of 210 users. The signatures were acquired in two sessions using a digitizing tablet. Each session contains 15 genuine signatures and 10 skilled forgeries per person.

- During the training phase, we used 5 randomly selected genuine signatures of each signer. During the test phase we used 10 genuine signatures and 10 so-called skilled forgeries [14] of each signer.

- Parameters of the algorithm had the following values: (a) the number of chromosomes in population $N$ pop $=100$, (b) the value of parameter $F$ [20] of the DE algorithm is 0.5 , (c) the value of parameter $C R$ [20] of the DE algorithm is 0.9 (see [20]), (d) the value of weights $w D_{i, j}$ is 0.7 , (e) the value of weights $w R_{i, j}$ is 0.2 , and (f) the value of the partition number parameter $P$ is 2 .

- The DE algorithm stopped after after 100 generations had been repeated.

- Identity verification of the test signature of the user claiming to be signer $i$ was performed using the flexible neuro-fuzzy one class classifier proposed by us in [4].

- The simulations were performed five times and the results were averaged.

- The test was performed using the authorial testing environment implemented in C\# language.

The conclusions from simulations can be summarized as follows
- The method proposed in this paper works according to the adopted assumptions, and the obtained accuracy is high (see Table 1).

- The accuracy of the proposed method is better than the accuracy of the method using a horizontal partitioning performed on the basis of the average values of the signals describing the signature dynamics. It confirms that horizontal partitions created on the basis of the values selected by a population-based algorithm with a properly defined fitness function contain more characteristic descriptors of the individual signature.

- The accuracy of the proposed method is better than the accuracy of other methods based on signature partitioning. It confirms that the use of a population-based algorithm with a properly defined fitness function can be used to determine more suited partitions that contain more characteristic descriptors for an individual user.

\section{Conclusions}

In this paper, we proposed a new method for the on-line signature verification. It uses possibilities offered by population-based algorithms. The purpose of the population algorithm is to generate dynamic signature partitions. In particular, it generates borderlines between partitions for the considered signals describing the dynamics of the signing process. Due to this, descriptors are created precisely, independently for each user. This means that the fuzzy system used to assess the genuineness of signatures can work with high accuracy. This 
was confirmed by the simulations for the BioSecure database.

In the future, we plan to test the use of the population algorithm for partitioning carried out in the time domain and partitioning combined with the prediction of changes in the boundary values between partitions.

\section{Acknowledgments}

The paper is financed under the program of the Polish Minister of Science and Higher Education under the name 'Regional Initiative of Excellence' in the years 2019-2022; project number 020/RID/2018/19; the amount of financing PLN $12,000,000.00$.

\section{References}

[1] Cpałka, K. Design of Interpretable Fuzzy Systems. Springer, Cham (2017)

[2] Cpałka, K., Zalasiński, M. On-line signature verification using vertical signature partitioning, Expert Systems with Applications, vol. 41, pp. 4170-4180 (2014)

[3] Cpałka, K., Zalasiński, M., Rutkowski, L. A new algorithm for identity verification based on the analysis of a handwritten dynamic signature, Applied Soft Computing, vol. 43, pp. 47-56 (2016)

[4] Cpałka, K., Zalasiński, M., Rutkowski, L. New method for the on-line signature verification based on horizontal partitioning, Pattern Recognition, vol. 47, pp. 2652-2661 (2014)

[5] Das, S., Suganthan, P.N. Differential evolution: A survey of the state-of-the-art. IEEE transactions on evolutionary computation, vol. 15(1), pp. 4-31 (2010)

[6] Dean, D., Sridharan, S. Dynamic visual features for audio-visual speaker verifi-cation, Comput. Speech Lang., vol. 24, pp. 136-149 (2010)

[7] Ekinci, M., Ayku, M. Human gait recognition based on kernel PCA using projec-tions, J. Comput. Sci. Technol., vol. 22, pp. 867-876 (2007)

[8] Faundez-Zanuy, M. On-line signature recognition based on VQ-DTW. Pattern Recogn. 40, 981-992 (2007)

[9] Fierrez-Aguilar, J., Nanni, L., Lopez-Penalba, J., Ortega-Garcia, J., Maltoni, D. An on-line signature verification system based on fusion of local and global information. Lecture Notes in Computer Science. Audio-and Video-based Biometric Person Authentication, vol. 3546, pp. 523-532 (2005)

[10] Fierrez, J., Ortega-Garcia, J., Ramos, D., Gonzalez-Rodriguez, J. HMM-based on-line signature verification: Feature extraction and signature modeling, Pattern Recognition Letters, vol. 28, pp. 2325-2334 (2007)

[11] Homepage of Association BioSecure. [Online] Available from: http://biosecure.it-sudparis.eu [Accessed: 13 May 2019]

[12] Houmani, N., Mayoue, A., Garcia-Salicetti, S., Dorizzi B., Khalil M.I., Moustafa, M.N., Abbas, H., Muramatsu, D., Yanikoglu, B., Kholmatov, A., Martinez-Diaz, M., Fierrez, J., OrtegaGarcia, J., Roure Alcobe, J., Fabregas, J., FaundezZanuy, M., Pascual-Gaspar, J.M., Cardenoso-Payo, V., Vivaracho-Pascual, C. BioSecure signature evaluation campaign (BSEC'2009): Evaluating online signature algorithms depending on the quality of signatures, Pattern Recognition, vol. 45, pp. 9931003 (2012)

[13] Ibrahim, M.T., Khan, M.A., Alimgeer, K.S., Khan, M.K., Taj, I.A., Guan, L. Velocity and pressurebased partitions of horizontal and vertical trajectories for on-line signature verification. Pattern Recogn. 43, 2817-2832 (2010)

[14] Jain, A.K., Ross, A. Introduction to Biometrics. In A.K. Jain, P. Flynn, A.A. Ross (Eds.), Handbook of Biometrics, Springer, Berlin-Heidelberg (2008)

[15] Kazikova, A., Pluhacek, M., Senkerik, R., Viktorin, A. Proposal of a new swarm optimization method inspired in bison behavior. In 23rd International Conference on Soft Computing, pp. 146-156, Springer, Cham (2017)

[16] Linden, J., Marquis, R., Bozza, S., Taroni, F. Dynamic signatures: A review of dynamic feature variation and forensic methodology, Forensic Science International, vol. 291, pp. 216-229 (2018)

[17] Łapa, K. Meta-optimization of multi-objective population-based algorithms using multi-objective performance metrics, Information Sciences, vol. 489, pp. 193-204 (2019)

[18] Mirjalili, S., Mirjalili, S.M., Lewis, A. Grey wolf optimizer. Advances in engineering software, vol. 69, pp. 46-61 (2014)

[19] Nanni, L., Maiorana, E., Lumini, A. and Campisi, P. Combining local, regional and global matchers for a template protected on-line signature verification system. Expert Systems with Applications, 37, 3676-3684 (2010) 
[20] Pedersen, M.E.H. Good parameters for differential evolution. Hvass Laboratories Technical Report, vol. HL1002 (2010)

[21] Prasad, M., Liu, Y.T., Li, D.L., Lin, Ch.T., Shah, R.R., Kaiwartya, O.P. A New Mechanism for Data Visualization with TSK-type Preprocessed Collaborative Fuzzy Rule based System, Journal of Artificial Intelligence and Soft Computing Research, vol. 7, 33-46 (2017)

[22] Riid, A., Preden, J.S. Design of fuzzy rule-based classifiers through granulation and consolidation, Journal of Artificial Intelligence and Soft Computing Research, vol. 7, pp. 137-147 (2017)
[23] Zalasinśki, M., Łapa, K., Cpałka, K. Prediction of values of the dynamic signature features, Expert Systems with Appications, vol. 104, pp. 86-96 (2018)

[24] Zalasinśki, M., Cpałka, K. A Method for Genetic Selection of the Dynamic Signature Global Features' Subset, Advances in Intelligent Systems and Computing, vol. 655, pp. 73-82 (2018)

[25] Zois, E.N., Alexandridis, A., Economou, G. Writer independent offline signature verification based on a symmetric pixel relations and unrelated trainingtesting data sets, Expert Systems With Applications, vol. 125, pp. 14-32 (2019)

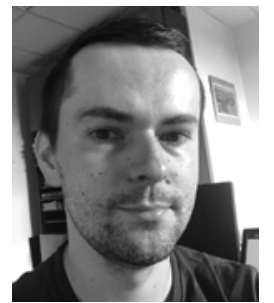

Marcin Zalasiński received the M.Sc. and Ph.D. degrees from the Częstochowa University of Technology, Częstochowa, Poland, in 2009 and 2014, respectively. He is currently an Associate Professor with the Department of Computer Engineering. Dr. Zalasiński has authored over 20 publications. His current research interests include computational intelligence, biometrics, and expert systems.

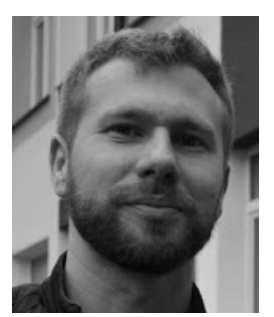

Krystian Lapa received the M.Sc. and Ph.D. degrees from the Częstochowa University of Technology, Częstochowa, Poland, in 2010 and 2015, respectively. He is currently an Associate Professor with the Department of Computer Engineering. Dr. Łapa has authored over 20 publications. His current research interests include computational intelligence, nature-inspired methods, and expert systems.

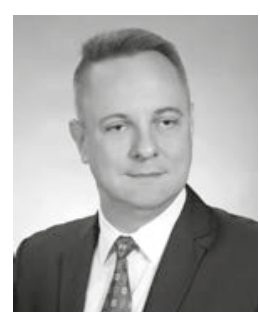

Krzysztof Cpalka received the M.Sc. and Ph.D. degrees from the Częstochowa University of Technology, Częstochowa, Poland, in 1997 and 2002, respectively. He is currently a Professor with the Department of Computer Engineering. Prof. Cpałka has authored over 100 publications, including several papers in various series of IEEE Transactions. His current research interests include computational intelligence methods and their applications. He was a recipient of the IEEE Transactions on Neural Networks Outstanding Paper Award in 2005 .

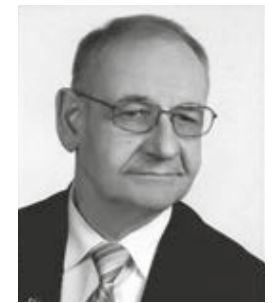

Krzysztof Przybyszewski is a professor at the University of Social Sciences in Łódź. His adventure with applied computer science began in the $1980 \mathrm{~s}$ with a simulation of non-quantum collective processes (the subject of a $\mathrm{Ph} . \mathrm{D}$. dissertation). At present, he is involved in research and applications of various artificial intelligence technologies and soft computing methods in selected IT problems (in particular, in expert systems supporting the management of education quality in universities - the use of fuzzy numbers and sets). As a deputy dean at the University of Social Sciences, he is the designer and organizer of the on-Computer Science Faculty education program. He is the author of over 80 publications in the field of computer science and IT applications.

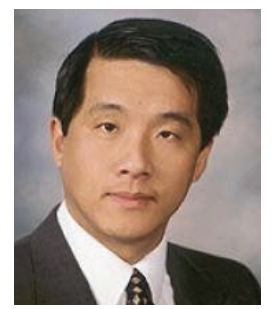

Gary G. Yen received his Ph.D. degree in electrical and computer engineering from the University of Notre Dame in 1992. He worked at the Structural Control Division of the USAF Research Laboratory in Albuqurque during 1992-1996. He is currently a Professor in the School of Electrical and Computer Engineering, Oklahoma State University in Stillwater. His research is supported by the DoD, DoE, EPA, NASA, NSF, and Process Industry. His research interest includes intelligent control, computational intelligence, conditional health monitoring, signal processing and their industrial/defense applications. Dr. Yen was an associate editor of the IEEE Control Systems Magazine, IEEE Transactions on Control Systems Technology, Automatica, Mechantronics, IEEE Transactions on Systems, Man and Cybernetics, Part A and Part B, IEEE Transactions on Neural Networks, and among others. He is currently serving as an associate editor for the IEEE Transactions on Evolutionary 
Computation and International Journal of Swarm Intelligence Research. He served as the General Chair for the 2003 IEEE International Symposium on Intelligent Control held in Houston and 2006 IEEE World Congress on Computational Intelligence held in Vancouver. Dr. Yen served as Vice President for the Technical Activities in 2005-2006 and President in 2010-2011 of the IEEE Computational Intelligence Society. $\mathrm{He}$ is the founding Editor-in-Chief of the IEEE Computational Intelligence Magazine. He received KC Wong Fellowship from the Chinese Acadamy of Sciences, Halliburton
Outstanding Faculty award, and OSU Regents Distinguished Research award. He also received an Honorary Professorship from Northeastern University, Sichuan University, and Dalian University of Technology in China. In 2011, he received the Andrew P Sage Best Transactions Paper award from the IEEE Systems, Man and Cybernetics Society. In 2013, he received Meritorious Service award from the IEEE Computational Intelligence Society. $\mathrm{He}$ is a distinguished lecturer from the IEEE Computational Intelligence Society, 20122014, an IEEE Fellow, and IET Fellow. 